\title{
Theoretical and Experimental Study of Radiation Pressure-Induced Mechanical Oscillations (Parametric Instability) in Optical Microcavities
}

\author{
H. Rokhsari, T. J. Kippenberg, T. Carmon, and K. J. Vahala
}

\begin{abstract}
Radiation pressure can couple the mechanical modes of an optical cavity structure to its optical modes, leading to parametric oscillation instability. This regime is characterized by regenerative oscillation of the mechanical cavity eigenmodes. Here, we present the first observation of this effect with a detailed theoretical and experimental analysis of these oscillations in ultrahigh- $Q$ microtoroids. Embodied within a microscale, chip-based device, this mechanism can benefit both research into macroscale quantum mechanical phenomena and improve the understanding of the mechanism within the context of laser interferometer gravitational-wave observatory (LIGO). It also suggests that new technologies are possible that will leverage the phenomenon within photonics.
\end{abstract}

Index Terms-Optical microcavities, optomechanical, parametric instability, photonic clock, radiation pressure.

\section{INTRODUCTION TO PARAMETRIC INSTABILITY}

A S CIRCULATING power is boosted in optical resonant systems, there will be a natural tendency for these systems to experience a radiation-pressure induced instability. The instability is manifested as regenerative oscillations of the mechanical modes of the cavity structure due to coupling of optical and mechanical degrees-of-freedom caused by radiation pressure. This has been recognized theoretically by V.B. Braginsky [1], [2], and is termed "parametric oscillation instability". At a simplistic level, this excitation process can be understood as follows. Each circulating photon in the cavity changes its propagation direction twice every roundtrip. Therefore, a photon transfers four times its linear momentum to the cavity's walls every time it completes a round trip. If the cavity is not infinitely rigid, the walls will deform in response to the resulting pressure. Hence, the pressure of circulating radiation induces a mechanical expansion of the cavity structure; this motion, in return, takes the optical cavity out of resonance with the input pump wave, thereby lowering the magnitude of radiation force. Upon restoration of the mechanical deformation, the process resumes, leading to a periodic motion of the cavity as well as the circulating power. It should be emphasized that this oscillation is regenerative, exhibiting classic threshold behavior and requiring no external modulation of the pump wave (see Fig. 1).

Manuscript received November 17, 2005; revised November 21, 2005. This work was supported by the National Science Foundation, Defense Advanced Research Projects Agency, Air Force Office of Scientific Research, and the Center for the Physics of Information.

The authors are with the Applied Physics Department, California Institute of Technology, Pasadena, CA 91106 USA (e-mail: vahala@caltech.edu).

Digital Object Identifier 10.1109/JSTQE.2005.862890

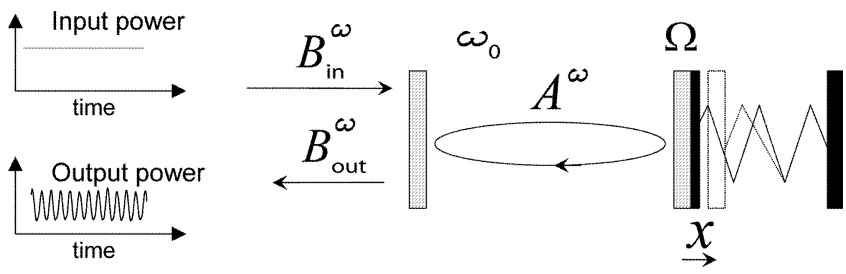

Fig. 1. Illustration of the radiation pressure-induced optomechanical coupling mechanism. $B_{\text {in }}^{\omega}$, the input optical field (at frequency $\omega$ close to a resonant frequency of the cavity $\omega_{0}$ ) to the Fabry-Perot causes large circulating field $A^{\omega}$ as a result of resonant power buildup in the cavity. The pressure caused by this power moves the free-to-move cavity wall by $x$, modeled as a damped harmonic oscillator at frequency $\Omega$. Motion of the end mirror, on the other hand, causes frequency change of the Fabry-Perot resonant optical mode. This interaction at sufficient optical powers results in regenerative oscillations of the end mirror and, consequently, the modulation of the output optical power $B_{\text {out }}^{\omega}$.

More precisely, if one assumes the optical pump-wave frequency $(\omega)$ is nearly resonant (but not exactly resonant) with the optical mode, radiation pressure-induced deformation of the cavity structure either lowers or raises the coupled optical pump power, depending upon the sign of detuning of the pump frequency relative to the cavity resonant frequency. It will be shown that when the pump laser is detuned to the high-frequency tail of the optical mode, the phase relationship between optical pressure and optical cavity deformation results in net power transfer from the optical pump to the mechanical mode. This transfer manifests itself mathematically as a gain for the mechanical oscillations, with a corresponding threshold optical pump-power.

Theoretical studies have been devoted to ramifications of this effect in the context of the Laser Interferometer GravitationalWave Observatory (LIGO) [3], [4]. It has been predicted during the past few years [1]-[6] that the parametric instability could limit the maximum stored energy in Fabry-Perot cavities (FP) used in the LIGO project, and hence limit the sensitivity of the gravitational wave detector. Although never observed, recently a bench top experimental setup was proposed to verify these theoretical concerns on a smaller scale [7].

As will be discussed in detail in the following, the mutual coupling of optical and mechanical modes is significantly enhanced in smaller size cavities. Whereas in macroscopic resonators the influence of radiation pressure is weak and only appreciable at high power levels [8], it is significant in optical microcavities (such as silica microspheres [9], microdisks, or microtoroids [10]) which simultaneously exhibit ultra-high- $Q$ optical modes and small mechanical mass. To give an example, when coupled to a waveguide, the high quality factor of whispering gallery 


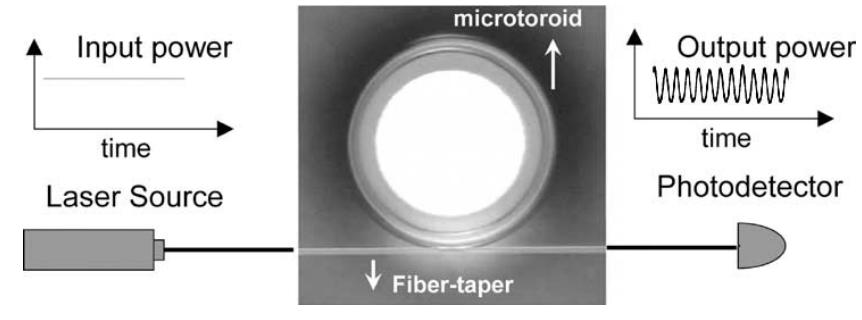

Fig. 2. Schematic of the simple experimental set up used for observation of transmission oscillations in toroid microcavities. The dc optical power of a laser source is coupled to a microtoroid through a tapered optical fiber. The transmitted optical power shows high amplitude modulations in the radio frequency range upon detection by a photodetector.

microcavities $\left(\sim 10^{8}\right)$ results in optical power buildup that can exceed $100 \mathrm{~W}$ of circulating power for only $1 \mathrm{~mW}$ of waveguide input power. The resulting radiation pressure due to confinement of these high optical powers in micron-scale volumes can expand the cavity structure such that the optical resonant frequency shifts by hundreds of the resonance linewidths. Therefore, the combination of high optical quality factor, small size, and small mechanical mass and dissipation can lead to threshold levels in the microwatt regime for regenerative mechanical oscillations (i.e., parametric oscillation instability) in whispering gallery microcavities.

Radiation-pressure-induced optomechanical interaction has similar properties to cavities containing a Kerr medium [11], including hysteretic wavelength response caused by radiation pressure [8]. In particular, both radiation pressure and the Kerr effect induce a coupling between optical path length and light intensity, one through cavity strain and the other through the index of refraction. It is, therefore, not surprising that radiation pressure has been proposed as an alternative path for observation of quantum effects such as squeezing [11] as well as numerous other quantum phenomena [12]-[17].

\section{INTRODUCTION TO TRANSMISSION OSCILLATIONS AND MECHANICAL EIGENMODES OF MICROTOROIDS}

In this work, we will focus on the radiation-pressureinduced parametric instability in whispering gallery microcavities, specifically microtoroid resonators [10]. However, all the aspects of this work may apply, in principle, to any type of optical cavity. The details of microtoroid fabrication process are reported in [10], but briefly, microtoroids are made of silica attached to a silicon cylindrical pillar. Microtoroids can support whispering gallery modes with $Q$ factors above 100 million, and can be directly coupled to tapered optical fibers.

We have recently observed transmission oscillations in the radio frequency (RF) range when optical power is coupled to sufficiently high quality factor optical modes $\left(Q \approx 10^{7}\right.$ or $10 \mathrm{~ns}$ photon lifetime at infra red wavelengths) of toroidal microcavities [18]-[20], see Fig. 2).

Spectral analysis of the detected transmitted optical power using a high-resolution electrical spectrum analyzer (ESA) revealed extremely narrow peaks (sub-Hertz linewidths) at a fre-

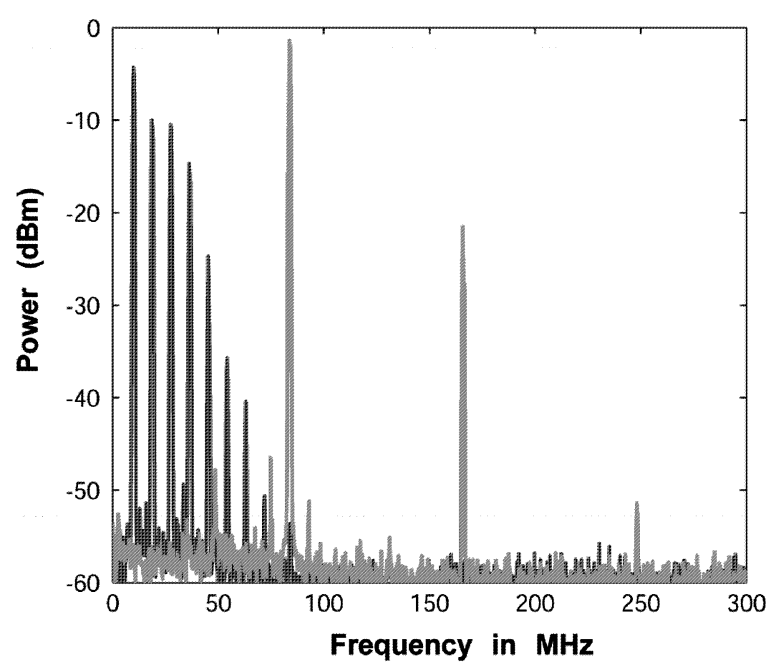

Fig. 3. The measured spectral content of pump power (at $1550 \mathrm{~nm}$ ) transmission as observed on an electrical spectrum analyzer (bandwidth set at $100 \mathrm{kHz}$ ). Two families of frequencies are observed, along with their harmonics. Those at lower frequency range are observed generally in the under-coupled regime, and the higher frequency oscillations mostly in the over-coupled regime.

quency typically in the range of $10-100 \mathrm{MHz}$, as well as at harmonics of this fundamental frequency (see Fig. 3). As can be seen in Fig. 3, typically two distinct fundamental oscillation frequencies (and their harmonics ${ }^{1}$ ) were observed: a low-frequency mode $(\sim 2-20 \mathrm{MHz})$, usually in the under-coupled regime, and a high-frequency mode $(\sim 40-100 \mathrm{MHz})$ in the over-coupled regime (see [21] and references therein for definition of under-, critical-, and over-coupled regimes).

Our further studies of this phenomenon revealed that in addition to their excellent optical properties, microtoroid resonators also exhibit high- $Q$ micromechanical modes, which are flexural or radial in character and exhibit experimentally observed $Q$-factors as high as 5000 . We numerically investigated the mechanical eigenmodes of a toroidal structure. The right panel of Fig. 4 shows the strain and stress of the first-three, rotationallysymmetric eigenmodes of a toroid microcavity obtained by finite element modeling. The left panel of Fig. 4 shows the experimentally observed oscillation frequencies plotted versus length $\mathrm{L}$ (see right panel for definition of $\mathrm{L}$ ) as inferred by scanning electron microscopy (SEM) imaging. As is evident, the frequencies increase with decreasing membrane length $(\mathrm{L})$. Close agreement of the measured RF oscillation frequencies with the results of the numerical modeling (less than $2 \%$ discrepancy) confirms that the first $(n=1)$ and third $(n=3)$ order flexural modes are responsible for generating the observed low- and high-frequency families of oscillations, respectively (see Fig. 3). In Section VI it will be explained why the $n=2$ mode is difficult to observe in our experiments.

\footnotetext{
${ }^{1}$ The characteristics of the overall waveguide-resonator system can be viewed as an optical modulator that is driven by this oscillation. This modulator has a nonlinear transfer function that manifests itself (in the modulated pump power) through the appearance of harmonics of the characteristic mechanical eigenfrequencies. These harmonics are easily observed upon detection of the modulated pump (see Fig. 2).
} 


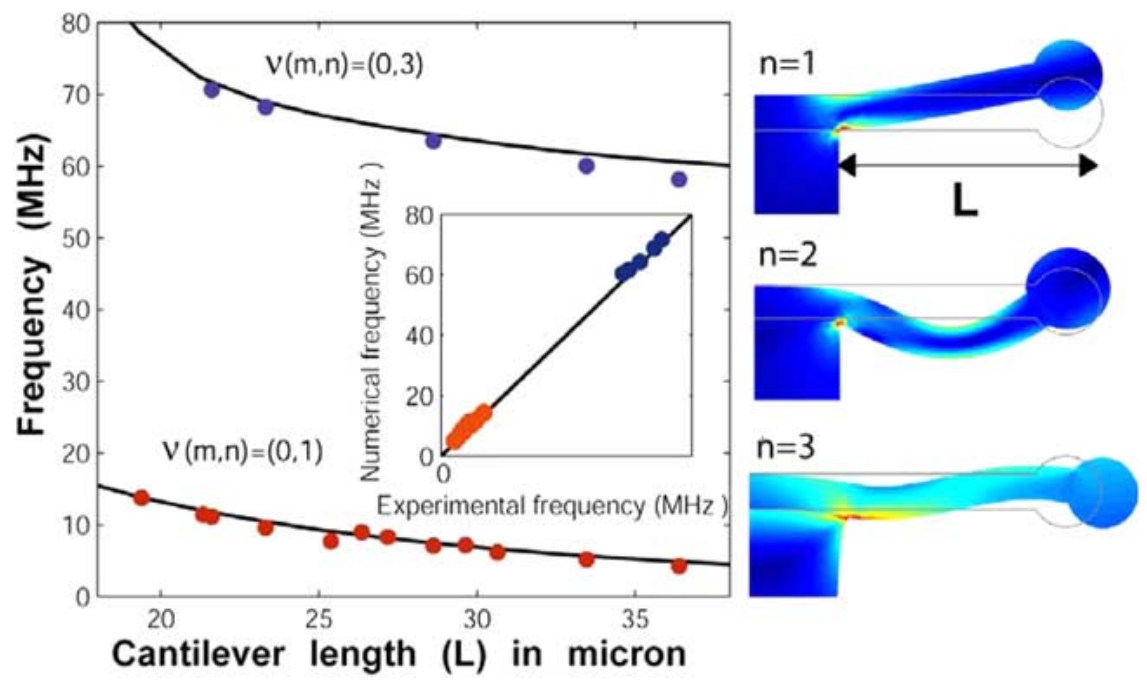

Fig. 4. Right panel: finite-element modeling of the micromechanical modes of a silica toroid microcavity. The radial and azimuthal mode order are denoted with $n$ and $m$ (where $m=0$ corresponds to rotationally symmetric modes). Shown are the first three rotationally symmetric radial modes $(n=1,2,3, m=0)$ in cross section with the amplitude of motion greatly exaggerated for clarity. In addition, the stress field is indicated using color. Note that the mechanical motion modulates the cavity path length due to a change in the cavity radius. Left panel: mechanical oscillation frequencies of the $(m=0, n=1)$ and $(m=0, n=3)$ modes versus the cantilever length $L$ (defined in the right panel). The dots are experimentally measured frequencies, and the solid lines are predictions of the numerical modeling. The inset shows the agreement between the numerical predictions of mechanical frequencies and measured frequencies of oscillations.

The mechanical origin of these oscillations was also confirmed by lowering a metallic microprobe into proximity with the plane of the silica disk connecting the toroid to the silicon pillar (i.e., not the toroid itself, where the optical mode lies). Since the probe is far removed from the toroidal whispering gallery, it affects only the mechanical and not the optical properties of the structure. The optical power oscillations were observed to fully quench upon probe contact.

As evident in the renderings provided in Fig. 4, the $n=3$ mechanical mode has a strong radial component to its motion, and hence understanding of its excitation by way of radiation pressure (which itself is primarily radial in direction) is straightforward. In contrast, the $\mathrm{n}=1$ mode motion is transverse, requiring a different method of force transduction (see Fig. 5). The details will be presented later in Section VI where it is shown that minute offsets of the optical mode from the equatorial plane provide a moment arm for action of radiation pressure. The resulting torque induces the transverse motion associated with the $n=1$ mode. Modeling and SEM measurement of the offset via focused ion beam preparation confirms this mechanism.

\section{EQUATIONS OF MOTION FOR OPTICAL AND MECHANICAL RESONATORS AND ADIABATIC APPROXIMATION}

This system can be modeled using a set of coupled differential equations, one governing the harmonic motion of the flexing toroid, and a second governing the resonant optical field. The equation of motion for deformation (more precisely, the displacement of the whispering gallery in the radial direction $(x)$ ) is that of a damped harmonic oscillator driven by radiation pressure (generated by the circulating optical field at the

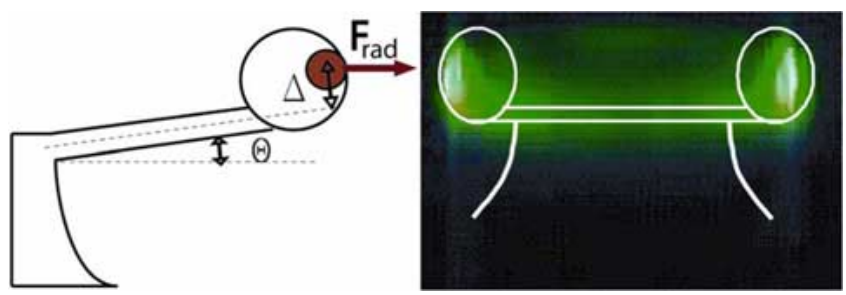

Fig. 5. Left panel shows the cross section and excitation mechanism of the fundamental $(n=1)$ flexural mode. The offset $(\Delta)$ between the optical mode location and the disk equatorial plane creates a lever arm for the radial optical force $\left(F_{\text {rad }}\right)$ and, subsequently, a torque. The right panel shows the side view image of a toroidal microcavity pumped by $\mathrm{CW}$ laser at $1550 \mathrm{~nm}$. Green luminescence is the result of Erbium up-conversion (from 1.5 to $0.5 \mu \mathrm{m}$ ), which in this specific case, is intentionally doped in the microtoroid to illustrate the location of the optical mode. Note that the optical mode (green) is higher than the "cantilever beam" holding the microtoroid, attesting the existence of the offset $(\Delta)$ in the left panel.

periphery of the microtoroid)

$$
x \ddot{(t)}+\gamma_{0} \dot{x}(t)+\Omega^{2} x(t)=\frac{f(t)}{m_{\mathrm{eff}}}=\frac{2 \pi n}{m_{\mathrm{eff}} c}|A(t)|^{2}
$$

where $m_{\mathrm{eff}}$ is the effective vibrating mass of the mechanical structure in the radial direction (the direction which alters the optical resonant frequencies of the cavity), $\Omega$ is the mechanical frequency of the oscillation (one of the eigenfrequencies of the structure), and $\gamma_{0}$ is the intrinsic mechanical damping coefficient determining the mechanical quality factor $Q_{m}=\Omega / \gamma_{0}$. $f(t)$ is the radial force applied by radiation pressure of the slowly varying field amplitude $A(t)$ (normalized so that $|A(t)|^{2}$ is the circulating optical power). $c / n$ is the velocity of light in the cavity. As the optical resonance shifts with structure expansion, the frequency difference between the input field and 
the moving-cavity resonance changes as

$$
\Delta \omega(t)=\Delta \omega_{0}-\left(\omega_{0} / R\right) x(t)
$$

Here, $R$ represents the radius of the microcavity (or more precisely of the optical whispering gallery mode). The mechanically induced displacement of the optical cavity resonant frequency contains, in general, a contribution from spatial and refractive index changes (stress optical effect) ${ }^{2}$ [22]. The optical field in the cavity, on the other hand, obeys the following equation [23]:

$$
\dot{A}(t)+A(t)\left[\frac{\omega}{2 Q_{\mathrm{tot}}}-i \Delta \omega(t)\right]=i B \sqrt{\frac{\omega}{T Q_{\mathrm{ext}}}}
$$

$B$ is the input pump field (normalized such that $|B|^{2}$ is optical power); $Q_{\text {total }}$ is the total quality factor of the optical mode, made up of an intrinsic contribution $Q_{0}$ and a waveguide-loading contribution $Q_{\text {ext }}: 1 / Q_{\text {total }}=1 / Q_{0}+1 / Q_{\text {ext }}$ (see [21]); and $T$ is the photon round trip time in the cavity.

In this section, we will solve the preceding system of equations assuming what we will call the "adiabatic approximation." This approximation holds when $\Omega \ll \omega / Q_{\text {tot }}$ (i.e., mechanical frequency is much smaller than the optical cavity bandwidth). The adiabatic picture provides a clear and intuitive understanding of the described optomechanical interaction. In Section V, we will solve the system with a more general approach, revealing the details of this interaction.

In the adiabatic regime, (1)-(3) can be solved selfconsistently, leading to a mechanical gain term which is a linear function of the circulating optical power, and that offsets intrinsic sources of mechanical damping as given as follows. This leads to a threshold optical pump power for onset of mechanical oscillations

$$
\begin{aligned}
\gamma & =\gamma_{1} 0\left(1-P / P_{\text {threshold }}\right), \\
P_{\text {threshold }} & =\frac{R^{2} \omega_{0}^{2} m \Omega}{64} f(d) \frac{Q_{\text {ext }}}{Q_{\text {total }}^{4} Q_{m}}
\end{aligned}
$$

where the intrinsic mechanical damping coefficient $\gamma_{0}$ is modified to $\gamma$ in the presence of the optical power in the waveguide $(P)$, and where $P_{\text {threshold }}$ denotes the incident (in the waveguide) threshold power (i.e., not the power coupled into the resonator).

At strong coupling regimes $\left(Q_{\text {ext }} \ll Q_{0}\right)$, the overall quality factor is dominated by waveguide loading (i.e., $Q_{\text {tot }} \approx Q_{\text {ext }}$ ), and hence (4) predicts that in this regime, the threshold power scales approximately with $1 / Q_{\text {total }}^{3}$, emphasizing the importance of high optical $Q$. Also apparent from (1) is the rapid scaling of the threshold power with cavity dimensions. Taking into account the scaling of the effective vibrating mass and its frequency, threshold power scales approximately with $P_{\text {threshold }} \propto R^{4}$. This explains why this effect, not yet observed in the context of the LIGO project, is more likely to occur in microcavities with high $Q$ factors and small dimensions.

\footnotetext{
${ }^{2}$ From the simulations, it is calculated that the change in refractive index due to the stress-optical effect is more than one order of magnitude smaller compared to the direct spatial change in cavity path length [i.e., $2 \pi(R+d R) \times(\Delta n+$ $\left.\left.n_{\text {eff }}\right) \approx 2 \pi n_{\text {eff }}(R+d R)\right]$.
}

In (4), $d$ is the detuning factor, giving the normalized detuning of the optical frequency from the resonant optical pump frequency of the cavity $\left(\omega_{0}\right)$ in units of its linewidth (i.e., $\left.d \equiv\left(\omega-\omega_{0}\right) /\left(2 \omega_{0} / Q_{\text {total }}\right)\right)$, and $f(d)=\left(1+d^{2}\right)^{3} / d$ is a factor that is positive for blue detuning of the pump (and negative for red detuning). This factor, emerging from the analysis, reflects the intuitive picture described earlier, requiring a particular phase relation between variation in coupled radiation pressure and cavity deformation in order that power is transferred from the optical mode to the mechanical mode, and not the other way. ${ }^{3}$ Analyzing the preceding system in the time domain by numerically solving the coupled differential (1)-(3) offers a clear picture of the energy flow between mechanical and optical fields, and the interaction between the mechanical and optical resonators both present in the same device. Applying the predictorcorrector Adam's method on (1)-(3) reveals the system dynamical behavior $(A(t), x(t))$. Having $A(t)$, one can calculate the output power $\left|\left(1-T \omega / 2 Q_{\mathrm{tot}}\right) B+i \sqrt{T \omega / Q_{\mathrm{tot}}} A(t)\right|^{2}$ [23]. Mechanical oscillations typically evolve to their full extent (starting from mechanical rest) within five time constants of the mechanical structure $(10 / \gamma)$. For small oscillation amplitudes $\left(x \ll R / Q_{\text {tot }}\right)$, a nearly linear amplitude relation exists between mechanical motion and output power modulation. For larger oscillations, however, the system response becomes nonlinear as the microstructure vibration amplitude induces a corresponding shift in optical resonant frequency that exceeds the cavity linewidth (see Fig. 6). Interference of the stationary pump wave and the field discharged from the cavity results in a train of decaying peaks reflecting the fact that light discharged from cavity is decaying exponentially with time, and is frequency shifted due to cavity vibrations. This behavior of output power is depicted in Fig. 6(a) and (b). At even larger oscillation amplitudes, we have also observed regimes where the system becomes sensitive to infinitesimal noise in the initial conditions when a specific threshold power is exceeded, resembling the chaotic behavior of the damped, driven pendulum.

Fig. 6 shows the numerically solved cavity deformation, the optical power in the cavity, and their interaction during a few mechanical time cycles. Cavity motion $r(t)$ (not to be confused with radial part of it $x(t)$ ) exhibits a flex of a few $\stackrel{A}{A}$, which corresponds to a resonance shift of few cavity bandwidths [Fig. 6(c)]. Cavity velocity $\left(\partial_{t} r(t)\right)$ is about $0.9 \mathrm{~mm} / \mathrm{s}$ when the cavity resonance crosses the pump laser wavelength [Fig. 6(d)]. The cavity is then charged to a circulating power $\left(|A(t)|^{2}\right)$ that approaches $50 \mathrm{~W}$ [Fig. 6(e)] causing a centrifugal radiation force $(f)$ that approaches $1.4 \mu N$. Upon cavity

\footnotetext{
${ }^{3}$ For $f(d)<0$, i.e., a red shift of the pump frequency with respect to the cavity mode, the phase of the radiation pressure variations actually damps or "cools" the vibrations. Note that no external feedback system is necessary here to damp the vibrations or "cool" the resonator. The feedback system is inherent to the coupling mechanism. Due to the high quality factor of our cavities $(Q \sim 10$ million) the "red shifted" tail of the optical mode is not thermally stable (see H. Rokhsari et al.,"Loss characterization in microcavities using the thermal bistability effect," Appl. Phys. Lett., vol. 85, pp. 3029-3031, 2004). Replacing the cavity material (silica) with a negative thermooptic coefficient material would stabilize the red shifted tail and cavity-cooling induced by radiation pressure effects could be observable.
} 

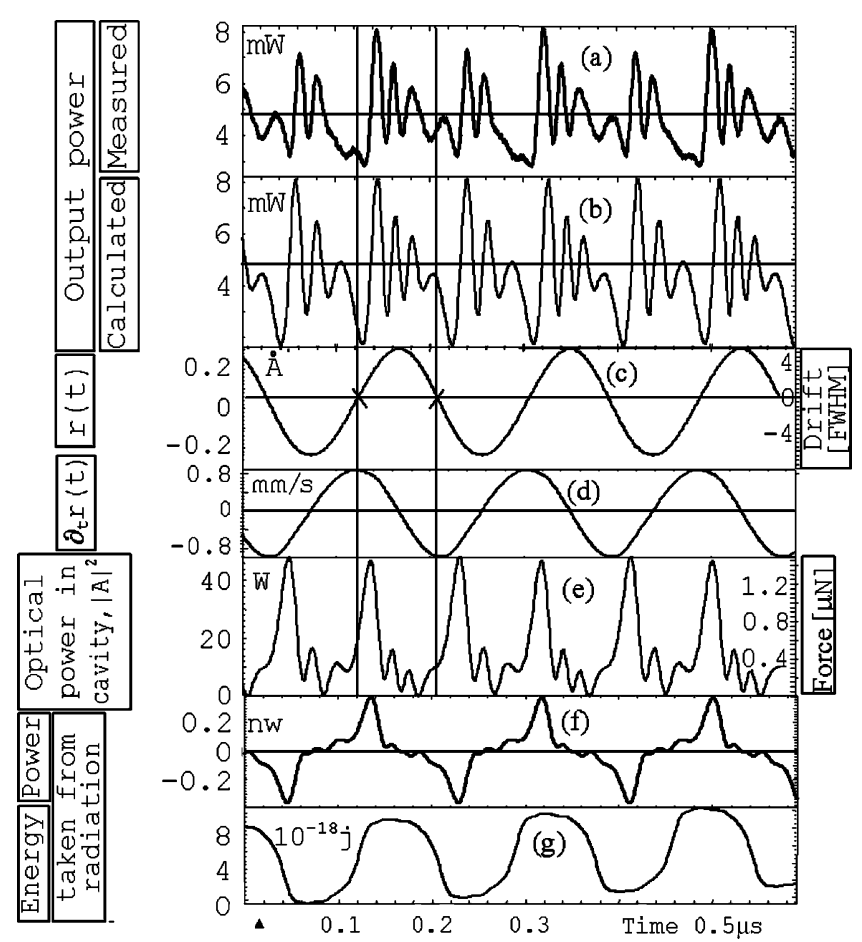

Fig. 6. Plots versus time of (a) measured, and (b) calculated transmitted optical power; (c) cavity displacement; (d) velocity; (e) optical power circulating in cavity (left ordinate) which is proportional to the total radial force (right ordinate) applied by radiation on the cavity; (f) power flow to mechanical mode; and (g) energy transferred to mechanical mode from radiation. Here, the toroid radius is $R=29 \mu \mathrm{m}$, the mass was calculated (from SEM imaging) to be $5 \times 10^{-11}$ $\mathrm{Kg}$, the intrinsic mechanical quality factor was measured to be 1200 , and the mechanical oscillation frequency occurs at $5.4 \mathrm{MHz}$. The pump power frequency is fixed at detuning of 0.55 full-width at half maximum (FWHM) away from the cavity optical resonance $\left(\lambda_{0}=1461 \mathrm{~nm}\right)$, and the optical quality factor was measured to be $Q_{o}=5 \times 10^{7}$.

expansion, the power $\left(f(t) \partial_{t} \mu r(t)\right)$ applied by radiation on the moving structure approaches $0.2 \mathrm{nW}$ [Fig. 6(f)]. The resulting transference of energy is manifested as a red Doppler shift in the circulating photons. Upon the cavity shrinking, power flows back to the optical mode from the mechanical potential energy; the circulating photons then experience a blue Doppler shift. A significant point is again the need for the pump wave to be blue detuned with respect to the microcavity resonance $(d>0$ in (4)) in order to induce oscillations. Because of this detuning, the pump wave spends a little more time to one side of the microcavity resonant frequency [Fig. 6(c)] explaining why energy $\left(\int_{0}^{t} f\left(t^{\prime}\right) \partial_{t^{\prime}} \mu r\left(t^{\prime}\right) d t^{\prime}\right)$ flows on average from the optical mode to the mechanical mode [Fig. 6(g)] and generates gain for mechanical vibrations as predicted by (4). In this example, for each acoustical cycle, $9 \times 10^{-18} \mathrm{~J}$ of energy are given to the optical mode upon shrinking, but then $10 \times 10^{-18} \mathrm{~J}$ are taken from the optical mode upon expansion [Fig. 6(g)]. This energy difference drives the mechanical vibrations. The sign of the energy flow will be reversed if the pump detuning changes sign. It is interesting, and the reader can verify, that the net energy transferred to the mechanical mode in every cycle $\left(10^{-18} \mathrm{~J}\right)$ is equal to the dissipated mechanical energy (stored mechanical energy divided by the mechanical quality factor, $\left.E_{\text {dissipated }}=\left(2 \pi E_{m}\right) /\left(Q_{m}\right)\right)$.

\section{EXPERIMENTAl Study OF BElow AND AbOVE THRESHOLD BEHAVIOR}

In order to verify the predictions of the preceding findings in the subthreshold regime, the mechanical damping rate was measured as a function of optical pump power. In this regime, damping of mechanical oscillations can be decreased by injecting optical power into the microtoroid, yet the induced mechanical gain is not sufficient to initiate the regenerative vibrations. To measure the damping rate, an optical pump and probe approach was used with two laser beams (a strong pump and a weak probe) individually resonant with two whispering gallery mode optical resonances. The experimental setup is depicted in Fig. 7.

Two, external-cavity diode lasers with $300 \mathrm{kHz}$ linewidth were used as laser sources. The pump laser in the $1550 \mathrm{~nm}$ band and the probe laser in the $1480 \mathrm{~nm}$ band were coupled to the microcavity using a tapered optical fiber formed by heating and stretching a length of single mode fiber [24]. The output of the $1550 \mathrm{~nm}$ laser was weakly modulated using a Mach-Zehnder modulator. In this way, the "dc" component of the pump power creates gain for the mechanical modes, while the alternating component creates a harmonically varying radiation pressure which acts as a forcing function on the subthreshold vibrational modes. Since the response motion also causes frequency shifts of the optical mode that are resonant with the probe signal, the mechanical response as a function of forcing-function frequency can be measured using the optical probe wave. The resulting modulation of the probe power can be measured using a lock-in amplifier, and it can be related to the amplitude of vibrations by measuring the quality factor of the optical mode coupled to the probe wave and its detuning from probe signal (for these experiments, the weak probe beam was coupled to a low $Q\left(\sim 10^{6}\right)$ whispering gallery resonance to ensure that probe power would not induce a competing oscillation effect). In this way, "vibrational spectroscopy" can be performed by sweeping the modulation frequency through the vibrational resonances. This spectroscopy reveals the lineshape of the mechanical resonance, and hence its linewidth (and damping rate or, equivalently, the mechanical $Q$ factor). Fig. 8 gives sample spectroscopic scans over an $n=1$ vibrational mode with a resonant frequency of $7.67 \mathrm{MHz}$. The solid lines are the theoretical fits using a damped harmonic oscillator model for the mechanical motion. The inset in Fig. 8 gives measured damping rates (extracted from the theoretical fits like that shown in the main figure) plotted versus optical pump power using the pump/probe spectroscopy measurements. A linear decrease in damping with increasing pump power is evident in the data (in agreement with (4)). Also apparent is the threshold power, which, in this case, is expected to occur at an optical pump power of $11 \mu \mathrm{W}$. The intrinsic $Q$ factor of the mechanical mode (i.e., $Q$ factor at zero input pump power) is inferred to be 630 in this data.

To characterize the mechanical modes in the above-threshold regime, the weak modulation of the pump power was terminated (i.e., steady pump wave) and the spectral content of the transmitted probe power was analyzed to monitor regenerative mechanical oscillation. The Fourier component of the transmitted probe 


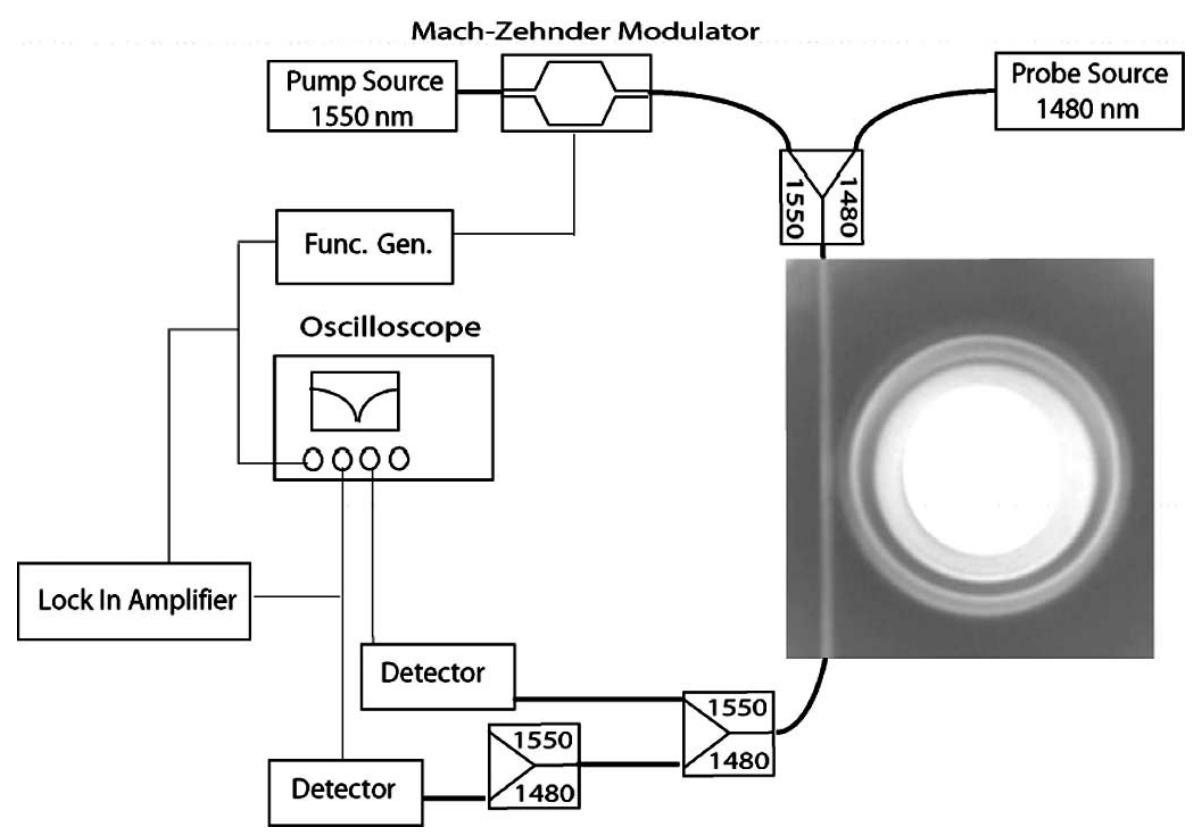

Fig. 7. The experimental setup used for characterizing the mechanical oscillations of microtoroids in the subthreshold regime. Two wavelength couplers are used to ensure high extinction of the pump power in the probe channel. The picture is a top-view optical micrograph of a toroid microresonator evanescently side coupled to a tapered optical fiber.

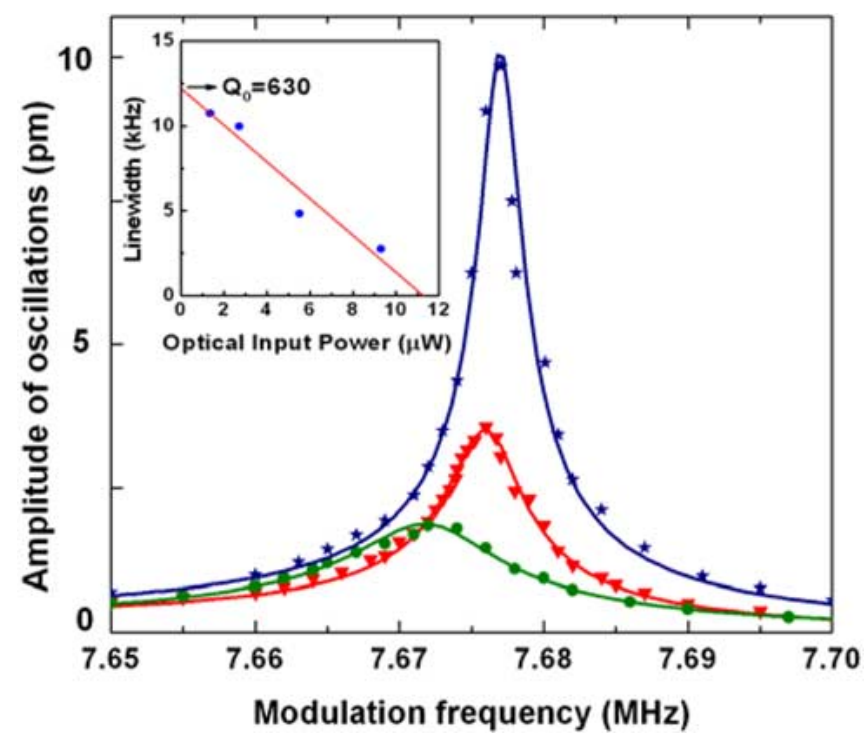

Fig. 8. Measured amplitude response (points) of the mechanical vibrations of an $n=1$ mechanical mode as a function of driving-force frequency (modulation frequency of the pump power). Circles (green), triangles (red), and stars (blue) represent the data for $2 \mu \mathrm{W}, 5 \mu \mathrm{W}$, and $9 \mu \mathrm{W}$ of average pump power. The inset shows the effect of the optical power on the linewidth of the mechanical oscillator inferred from the theoretical fits (such as the solid lines in the main figure). A linear fit shows a threshold of $11 \mu \mathrm{W}$ for the mechanical oscillations, and an intrinsic quality factor of 630 for the measured mechanical mode of the toroidal structure.

power at the mechanical resonant frequency $(\Omega)$ was monitored by the electricalspectrum analyzer. The intensity of this signal, proportional to the amplitude of the vibrations caused by the pump wave, was measured as a function of pump power. Fig. 9 contains a typical result of these measurements for one of the studied devices, and shows a clear threshold for the vibrational

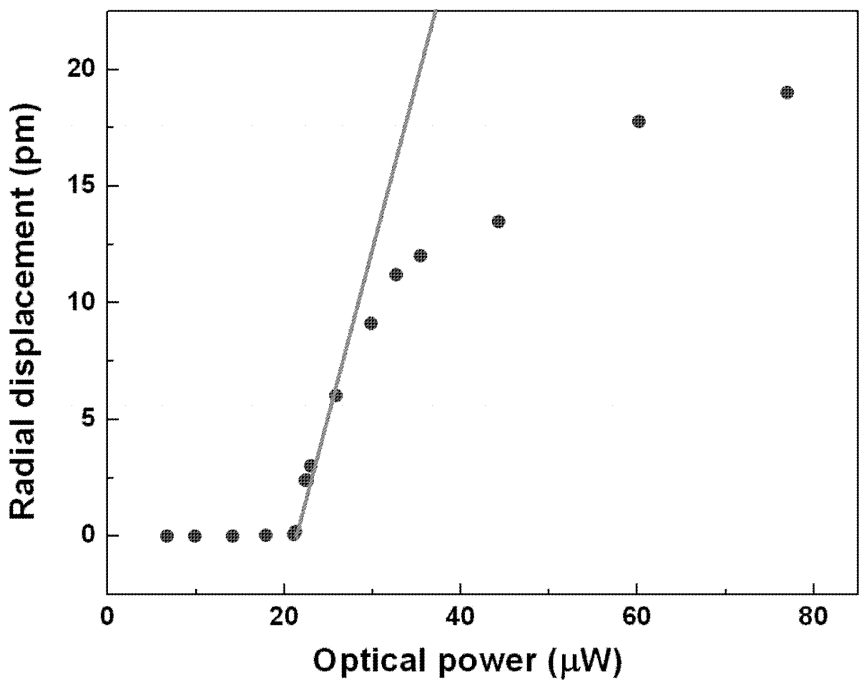

Fig. 9. Measured mechanical oscillator displacement as a function of the optical pump power showing threshold behavior. Oscillations initiate at about $20 \mu \mathrm{W}$ of input power, and start to saturate for higher values of pump power. This saturation is associated with the lower optical-mechanical coupling at displacements large enough to shift the resonant frequency of the optical mode by greater than its linewidth.

oscillations. Since the oscillator behaves as an optical power modulator $^{1}$, the electrical spectrum analyzer data can be transformed to compute the actual amplitude of mechanical motion. More precisely, modulation depth in the optical power can be transformed into frequency variation caused by the mechanical motion using the measured linewidth of the optical mode. This frequency variation, however, is directly proportional to the ratio of amplitude of the mechanical motion to the radius of the microtoroid. This transformation has been used to calibrate 


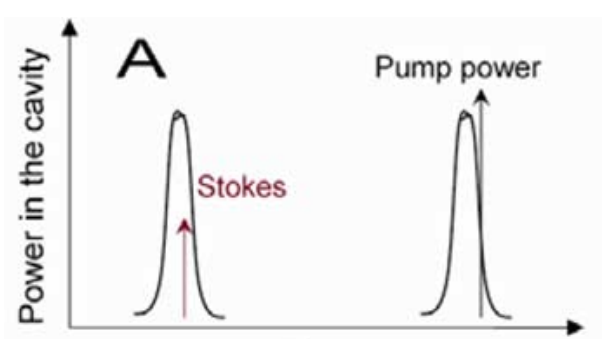

Frequency

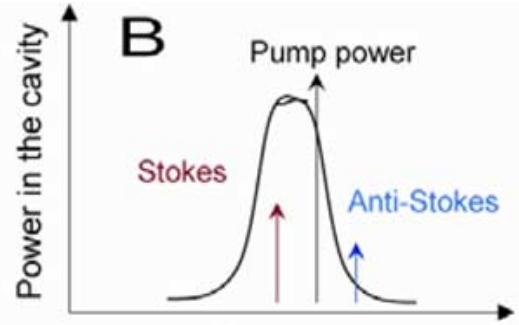

Frequency

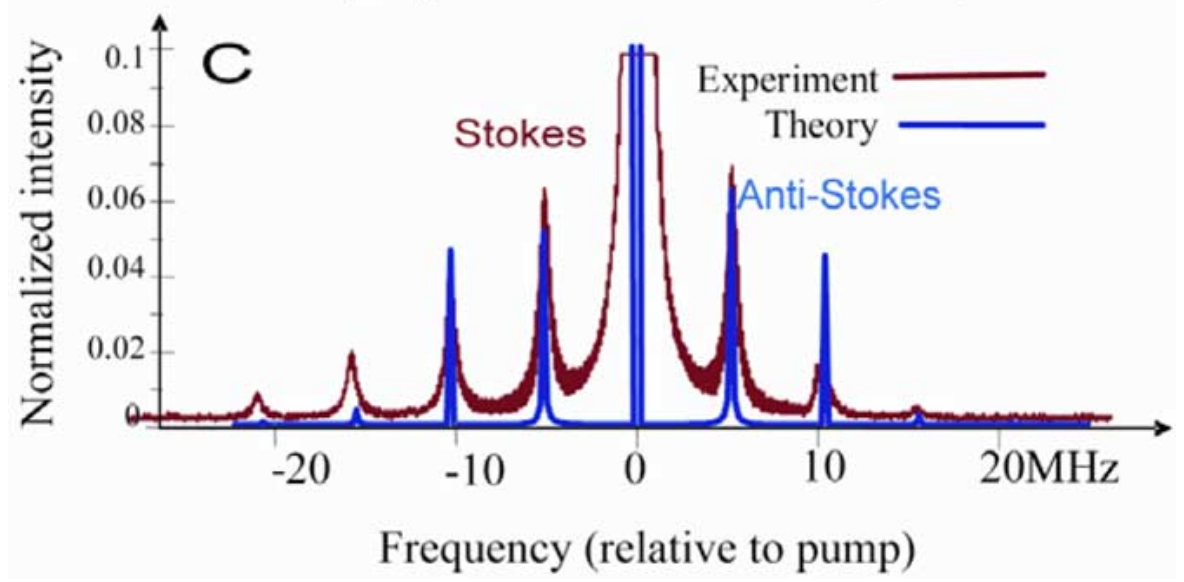

Fig. 10. Panel A represents Braginsky's view of parametric instability in the frequency domain, where the Stokes sideband coincides with an adjacent optical mode. Panel B shows the scenario we have encountered in our observations where the Stokes and anti-Stokes sidebands both fall almost within the bandwidth of the same optical mode. Panel C shows the experimental demonstration of the panel B scenario. Optical power out-coupled from a toroid microcavity oscillating at about 5-MHz frequency is measured by a 1-MHz-resolution spectrum analyzer (high finesse Fabry-Perot). Higher order optical sidebands exist due to the nonlinear nature of the "modulation" transfer response function of the resonator-waveguide system. Theoretical predictions are also given.

the vertical axis in Fig. 9. The data in Fig. 9 also seem to suggest that the amplitude of the vibrations saturates at high pump powers. Numerical modeling shows that this behavior can be attributed to the induced frequency shifts of the cavity which, for higher pump power levels, exceed the cavity linewidth. This, in turn, reduces the efficacy of the pumping mechanism as the pump wave spends a progressively smaller fraction of time on resonance during each mechanical cycle. ${ }^{4}$

\section{Beyond Adiabatic Approximation: Coupled Mode THEORY OF PARAMETRIC INSTABILITY}

In the theoretical work of Braginsky [1], [2], it was shown that the interaction of the vibrating resonator (at frequency $\Omega$ ) with photons inside the cavity results in creation of photons down-shifted (Stokes sideband, $\omega-\Omega$ ) or up-shifted (antiStokes sideband, $\omega+\Omega$ ) in energy from the original photons by the frequency of the vibrations. If the Stokes field coincides with an adjacent optical resonance [Fig. 10(a)], beating of the pump and Stokes sideband provides mechanical gain for regenerative mechanical oscillations, thereby causing the parametric oscillation instability.

It is important to note that optical resonances with $Q$-factors in the $10^{6}-10^{8}$ range have resonant linewidths in the range of approximately 2-200 MHZ in the infrared frequencies, which

${ }^{4}$ For the sample tested, it is calculated that radial variations of about $10 \mathrm{pm}$ will shift the resonant frequency of the excited optical mode by its linewidth. is indeed the range of the first three fundamental flexural modes for typical toroidal geometries employed in this work. This insight suggests that in addition to the Braginsky's theory [1], where the Stokes mode must coincide with an adjacent optical mode, mechanical oscillations can also occur when the mechanical resonance frequencies $(\Omega)$ falls within the same cavity bandwidth $\left(\omega / Q_{\mathrm{tot}}\right)^{5}$ of the pump mode (i.e., $\left.\Omega \approx \omega / Q_{\mathrm{tot}}\right)$, henceforth called the "in-band" case [Fig. 10(b)].

The adiabatic regime, for example $\Omega \ll \omega / Q_{\text {tot }}$ (see Section III), is, in fact, a special case of the in-band parametric instability $\left(\Omega \approx \omega / Q_{\text {tot }}\right)$, where Braginsky's "out of band" picture does not hold. The adiabatic approach has to be modified, however, to predict correct threshold powers where the mechanical frequency is comparable or even larger than the optical resonant linewidth (which are also observed in our studies), but is still too small to generate sidebands coinciding with an adjacent optical mode [see Fig. 10(a) and (b)]. To arrive at an analytical expression for the threshold power in these cases, we have extended the theory of Bragisnky for a Fabry-Perot cavity [1]. Using the slowly varying envelope approximation and the formalism of H. Haus, the mutual coupling of mechanical

\footnotetext{
${ }^{5}$ In special cases, we also observed the classical V. B Braginsky case, which can occur since the WGM modes typically appear as doublets, due to the lifting of the $\mathrm{CW}$ and $\mathrm{CCW}$ mode degeneracy. If this doublet splitting coincides with the mechanical oscillation frequency, parametric oscillation instability can occur, when pumping the blue-shifted doublet eigenmode, whereas it is absent on the red-shifted eigenmode.
} 
and optical modes can be described by

$$
\begin{aligned}
\frac{d}{d t} x_{m}= & -\frac{\Omega}{2 Q_{m}} x_{m}-\frac{i}{2 R n_{\mathrm{eff}} \sqrt{m_{\mathrm{eff}}} C(\Gamma)} e^{i \Omega t} \cdot|a|^{2} \\
\frac{d}{d t} a= & -\frac{\omega_{0}}{2 Q_{\mathrm{tot}}} a+\left[i \Delta \omega+\frac{i \omega_{0}}{R \Omega \sqrt{m_{\mathrm{eff}}}} e^{i \Omega t} x_{m}\right] a \\
& +i \sqrt{\frac{\omega_{0}}{Q_{\mathrm{tot}}}} B .
\end{aligned}
$$

These equations are identical to (1)-(3). The first equation describes the mechanical eigenmode with an effective mass $m_{\text {eff }}$, where $x_{m}$ (as opposed to $x$, which represents the radial motion of the cavity used in (1)-(3)) is normalized to mechanical energy; i.e., $\left|x_{m}\right|^{2}=\sum_{i=r, z, \theta} \int \varepsilon_{i} \sigma_{i} d V\left(\sigma_{i}\right.$ and $\varepsilon_{i}$ are the diagonal components of the stress and strain tensor), which decays with the lifetime $\left(Q_{m}\right) /(\Omega) . C(\Gamma)$ is a correction factor $[1, \ldots, 2]$ due to the reduction of circulating power in the presence of modal coupling [25]. Correspondingly, is the energy in the optical WGM mode (as opposed to $|A(t)|^{2}$, used previously to denote the optical power in the cavity), which is excited with a pump laser (with power $|B|^{2}$ ) detuned by the amount $\Delta \omega$ from line-center, $\Delta \omega=\omega-\omega_{o}$.

Assuming the optical field in the cavity is composed of the pump, and the generated Stokes and anti-Stokes fields with corresponding detunings $\Delta \omega, \Delta \omega_{S}=\Delta \omega-\Omega$, and $\Delta \omega_{\mathrm{AS}}=$ $\Delta \omega+\Omega$, from cavity line-center $\omega_{0}$ (i.e., $a e^{i \Delta \omega t}=a_{p} e^{i \Delta \omega t}+$ $a_{S} e^{i \Delta \omega_{S} t}+a_{\mathrm{AS}} e^{i \Delta \omega_{\mathrm{AS}} t}$ ), then (5) results in four coupled mode equations

$$
\begin{aligned}
\frac{\partial x_{m}}{\partial t}= & -\frac{\Omega}{2 Q_{m}} x_{m}+\frac{-i}{2 R n_{\mathrm{eff}} \sqrt{m_{\mathrm{eff}}} C(\Gamma)}\left(a_{p}^{*} a_{\mathrm{AS}}+a_{p} a_{S}^{*}\right), \\
\frac{\partial a_{p}}{\partial t}= & -\frac{\omega}{2 Q_{\mathrm{tot}}} a_{p}+i \Delta \omega a_{p} \\
& +\frac{i \omega}{R \sqrt{m_{\mathrm{eff}}} \Omega}\left(x_{m}^{*} a_{\mathrm{AS}}+x_{m} a_{S}\right)+i \sqrt{\frac{\omega}{Q_{\mathrm{ext}}}} B \\
\frac{\partial a_{S}^{*}}{\partial t}= & -\frac{\omega}{2 Q_{\mathrm{tot}}} a_{S}^{*}-i(\Delta \omega-\Omega) a_{S}^{*}-\frac{i \omega}{R \sqrt{m_{\mathrm{eff}}} \Omega} x_{m} a_{p}^{*}, \\
\frac{\partial a_{\mathrm{AS}}}{\partial t}= & -\frac{\omega}{2 Q_{\mathrm{tot}}} a_{\mathrm{AS}}+i(\Delta \omega+\Omega) a_{\mathrm{AS}}+\frac{i \omega}{R \sqrt{m_{\mathrm{eff}}} \Omega} x_{m} a_{p} .
\end{aligned}
$$

Solving (6) in steady state yields the threshold for radiation pressure-induced mechanical oscillation

$$
\begin{aligned}
P_{\text {thresh }}= & R^{2} m_{\mathrm{eff}} \frac{\omega_{0}}{Q_{0} Q_{\mathrm{tot}}} \frac{\Omega^{2}}{Q_{m}} \frac{\left|1+K+2 i Q_{0} \Delta \omega / \omega_{0}\right|^{2}}{8 K} \\
& \times\left[\frac{1}{1+4\left(\frac{Q_{\mathrm{tot}} \Delta \omega_{\mathrm{AS}}}{\omega_{o}}\right)^{2}}-\frac{1}{1+4\left(\frac{Q_{\mathrm{tot}} \Delta \omega_{S}}{\omega_{o}}\right)^{2}}\right]^{-1} .
\end{aligned}
$$

The threshold equation reveals that in order to arrive at oscillation, the mechanical loss has to be overcome (i.e., the expected $(1) /\left(Q_{m}\right)$ dependence). Dependence of radiation pressure upon

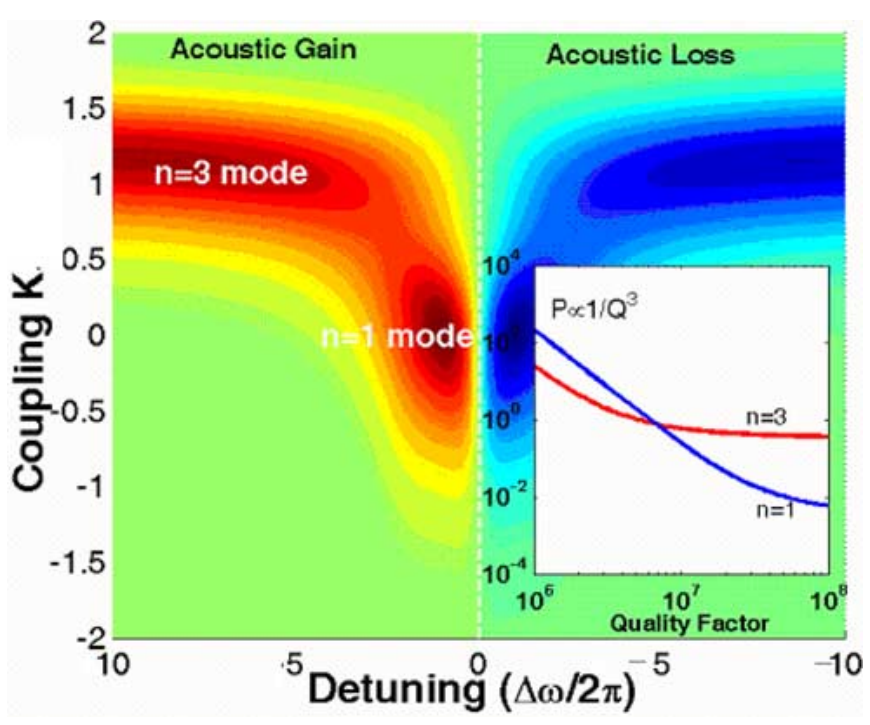

Fig. 11. Mechanical gain as a function of detuning (negative detuning corresponds to a redshift) and taper waveguide cavity-coupling (simulation parameters $v_{1}=4.4 \mathrm{MHz}, v_{3}=49.8 \mathrm{MHz}, Q_{0}=5 \times 10^{7}$ ). Maximum gain for the $n=1$ mode occurs in the undercoupled regime $(\log (K)<0)$, whereas for the $n=3$ mode it occurs overcoupled $(\log (K)>0)$. Inset: $\log -\log$ plot of the minimum oscillation threshold power for the $n=1$ and $n=3$ mode (7) versus the intrinsic optical quality factor.

circulating optical power, however, leads to the $(1) /\left(Q_{0}\right)$ dependence, as well as the presence of a weighting factor describing the effect of waveguide coupling, $K=\left(Q_{o}\right) /\left(Q_{\mathrm{ex}}\right)$ [25]. The last term results from the interplay of the Stokes and anti-Stokes photons and is related to the detuning factor $f(d)$ introduced in Section III on the adiabatic approximation. Fig. 11 shows a plot of the mechanical gain as a function of both coupling $(\mathrm{K})$ and detuning frequency $(\Delta \omega)$ for the $n=1$ and $n=3$ mechanical modes, which will be predominantly analyzed in the experiments. It is noted that the mechanical gain is only positive for a blue detuned pump with respect to the cavity resonance $\Delta \omega>0$. This situation leads to more Stokes than anti-Stokes photons (the ratio being $\left|\left(a_{\mathrm{AS}}\right) /\left(a_{S}\right)\right|^{2}=\left(\left(\omega_{0} / Q_{\mathrm{tot}}\right)^{2}+4\left(\Delta \omega_{S}\right)^{2}\right) /$ $\left.\left(\left(\omega_{0} / Q_{\text {tot }}\right)^{2}+4\left(\Delta \omega_{\mathrm{AS}}\right)^{2}\right)\right)$, and causes a net mechanical gain. The converse is true for red-detuned pump, which causes the mechanical resonance to experience negative gain (i.e., damping). For the special case of zero pump detuning, the gain vanishes. These predictions are in agreement with our findings in the adiabatic regime (see (4) and definition of $d$ factor).

Furthermore, it is interesting to investigate the exact $Q$-factor dependence of threshold power shown in the inset of Fig. 11. As expected, in the adiabatic regime $\Omega \ll$ $\omega / Q_{\text {tot }}$, the mechanical oscillation threshold power scales as $P_{\text {thresh }} \propto(1) /\left(Q_{m}\right) \cdot\left((1) /\left(Q_{0}\right)\right)^{3}$ in agreement with our previous expression for this regime (the reader can verify that in this regime, the expression for threshold power reduces exactly to (4)). In contrast, if the mechanical eigenfrequencies lie outside the cavity bandwidth, i.e., $\Omega>\omega / Q_{\text {tot }}$, then the threshold scales as $P_{\text {thresh }} \propto(1) /\left(\Omega^{2} Q_{m}\right) \cdot(\Delta \omega) /\left(\omega_{0}\right)$, causing a rolloff of the optical $Q$ dependence. In fact, analysis of (7) shows that the minimum threshold $\left(\left(\partial P_{\text {thresh }}\right) /(\partial K \partial \Delta \omega)=0\right)$ for higher optical $Q$ factors approaches asymptotically a limiting 


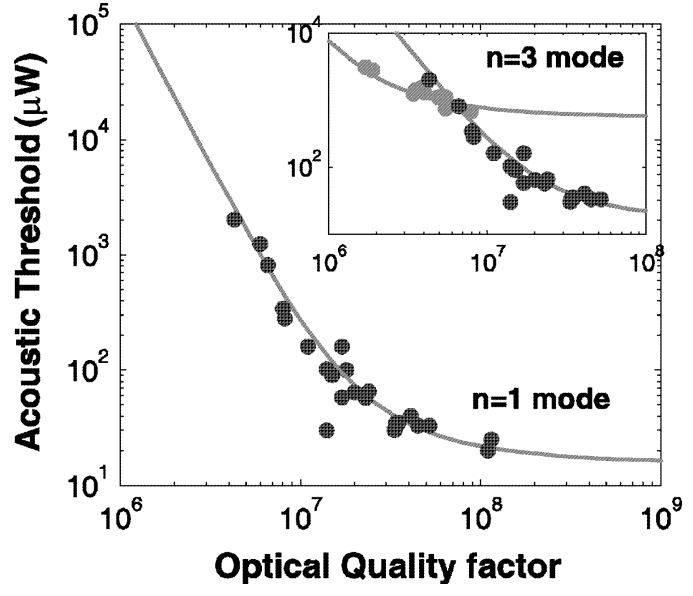

Fig. 12. Main panel: measured mechanical oscillation threshold $(\mu W)$ plotted versus the optical quality factor for the fundamental flexural mode $(n=$ $\left.1, \Omega / 2 \pi=4.4 \mathrm{MHz}, Q_{m}=3500\right)$ on a double logarithmic scale. The solid line is a one-parameter theoretical fit obtained from the minimum threshold equation by first performing a minimization with respect to coupling and pump wave detuning, and then fitting by adjustment of the effective mass $\left(m_{\text {eff }}^{(1)} \approx 3.3 \times 10^{-8} \mathrm{Kg}\right)$. Inset shows the measured threshold for the 3 rd order mode ( $\left.n=3, \Omega / 2 \pi=49.8 \mathrm{MHz}, Q_{m}=2500\right)$ plotted versus optical $\mathrm{Q}$. The solid line gives again the theoretical prediction with $m_{\text {eff }}^{(3)} \approx 5 \times 10^{-11} \mathrm{~kg}$. The $n=1$ mode data from the main figure is superimposed for comparison.

value, which can only be achieved for progressively stronger overcoupling (and correspondingly increasing the cavity bandwidth until the condition $\omega / Q_{\text {tot }} \approx \Omega$ is met). The rollover from inverse-cubic behavior occurs when $\left(\omega_{0}\right) /\left(Q_{0}\right) \propto \Omega$; i.e., $Q_{0}^{\text {roll-over }} \cong\left(\omega_{0}\right) /(\Omega)$ as observed in the inset of Fig. 11 .

\section{EXPERIMENTAL INVESTIGATION OF PARAMETRIC INSTABILITY THRESHOLD POWER}

In order to confirm the threshold dependence (as given by (7)) on both optical and mechanical $Q$-factors, we have carried out numerous experiments and simulations on a single toroid microresonator. The microtoroid under consideration had principal, pillar, and toroid diameters of $72 \mu \mathrm{m}, 36 \mu \mathrm{m}$, and $6.8 \mu \mathrm{m}$ and possessed mechanical resonance frequencies at $(4.4 \mathrm{MHz}, 25.6 \mathrm{MHz}$, and $49.8 \mathrm{MHz})$ for the first three modes $(n=1,2,3$ and $m=0)$. Minimum threshold power was measured by optimizing the detuning and coupling (compare Fig. 11). The result of this measurement is shown in Fig. 12. A Log-Log plot is used to infer the critical exponent, which shows excellent agreement with the prediction $\left(1 / Q^{3}\right)$ for the adiabatic regime. For progressively higher $Q$ values, the theoretically predicted rollover of the $1 / Q^{3}$ threshold dependence is observed. The rollover point occurs at a $Q$ of approximately $10^{7}$, which agrees well with the prediction $Q_{0}^{\text {roll-over }}=\left(\omega_{0}\right) /(\Omega)$ for the first-order flexural mode. The solid line is the minimum threshold as given by (7) which is minimized with respect to detuning $(\Delta \omega)$ and coupling $(\mathrm{K})$ to reflect the experiments; i.e., $\left(\partial P_{\text {thresh }}\right) /(\partial K \partial \Delta \omega)=0$. The effective mass was the only fit parameter used, and was inferred to be $\left(m_{\mathrm{eff}}^{(1)} \approx 3.3 \times 10^{-8} \mathrm{~kg}\right)$.

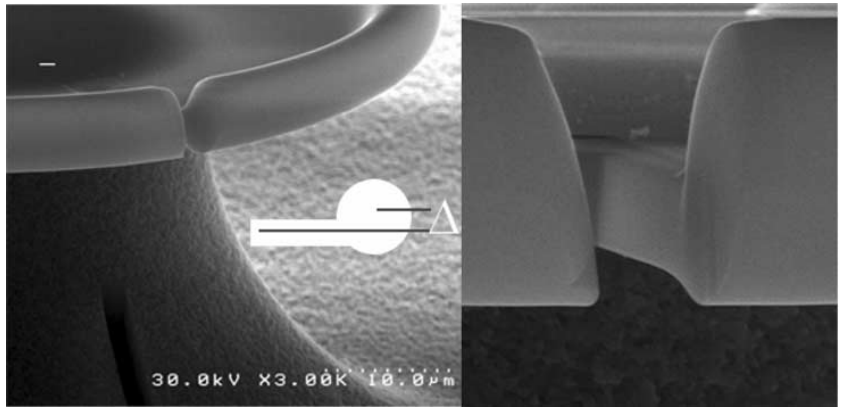

Fig. 13. Scanning electron micrograph of a cross section of the toroid microcavity used in this study. The cross section was revealed by focused ion beam (FIB) technology, which allowed removal of a $2-\mu$ m-wide section. The slicing clearly reveals the presence of an offset of the $7-\mu \mathrm{m}$-diameter toroid with respect to the $2-\mu \mathrm{m}$-thick silica support disk (in this case, an offset of approximately $1.3 \mu \mathrm{m}$, dotted lines). Inset: side view of the toroid microcavity inferred from the FIB method.

The threshold for the third-order flexural mode was also recorded. This mode has a resonance frequency of $49.8 \mathrm{MHz}$ with $Q_{m}^{n=3} \approx 2500$. Consequently, for optical $Q$ factors in the range of $10^{7}$, this mode is in the beyond-cavity-bandwidth regime, since $Q_{0}^{\text {roll-over }}=\left(\omega_{0}\right) /(\Omega)=4 \times 10^{6}$. In the experiments, oscillation on this high frequency mode was only observed by adjusting the taper-resonator coupling junction into the over-coupled regime, in agreement with theory. In this regime, the low frequency flexural mode could not be excited any more, and a transition from $n=1$ to $n=3$ occurred. This transition is in excellent agreement with the theoretical prediction of (7), as plotted in Fig. 11. The inset of Fig. 12 shows the measured threshold for the $n=3$ mode in comparison with the $n=1$ mode, both plotted versus optical $Q$. The single parameter fit yields an effective mass for the $n=3$ mode which is significantly lower than for $n=1$ by a factor of 660 ; i.e., $m_{\text {eff }}^{(3)} \approx 5 \times 10^{-11} \mathrm{~kg}$. Note that this measured dependence should be compared to the theoretical plot given within the inset to Fig. 11.

For the $n=3$ mode, the predicted effective mass from our numerical models was $m_{\mathrm{eff}}^{(3)} \approx 5 \times 10^{-11} \mathrm{~kg}$, which is in very good agreement with the experimental fit in Fig. 12. Furthermore, this value is exceptionally close to the actual mass of the vibrating structure.

For the $n=1,2$ modes, however, the calculated effective mass is a strong function of the offset of the toroidal ring with respect to the equatorial plane of the disk. ${ }^{6}$ As explained in Section II, excitation of the $n=1$ mode by radiation pressure can only be explained by existence of this offset. To both validate and quantify this offset, a cross section of the toroid microcavity used in the study was obtained with focused-ion beam slicing. SEM imaging, included in Fig. 13, reveals the presence of the previously postulated equatorial offset, which amounts to an offset of $1.3 \mu \mathrm{m}$. Incorporation of this offset to the numerical mass calculations yields $m_{\mathrm{eff}}^{(1)} \approx 2.6 \times 10^{-8} \mathrm{~kg}$, and

\footnotetext{
${ }^{6}$ We note that such an offset must exist, since otherwise mechanical motion of the $n=1,2$ modes causes modulation of the path length at the frequency $2 \Omega$ (i.e., frequency doubling), which is not observed in experiments.
} 


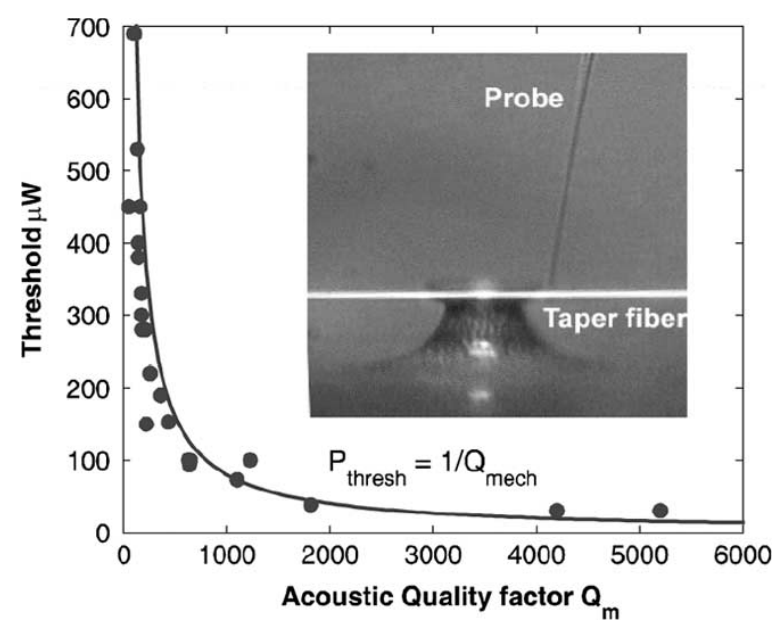

Fig. 14. Mechanical threshold power (in $\mu \mathrm{W}$ ) versus the mechanical quality factor of the $n=1$ mode. The solid line is the theoretical prediction $\left(P_{\text {thresh }} \propto\right.$ $\left.1 / Q_{m}\right)$. Inset: optical micrograph of the side view of the experimental setup, consisting of a silica microprobe in proximity to a fiber-coupled microtoroid of $72 \mu \mathrm{m}$ principal diameter.

$m_{\text {eff }}^{(2)} \approx 2 \times 10^{-9} \mathrm{Kg}$. The $n=1$ value agrees very well with the experimental values from Fig. 12. Finally, the numerical model also explains why the $n=2$ mode is observed only subthreshold in the experiments. The low mechanical $Q$ value $(\sim 200)$, in conjunction with its high effective mass and frequency, predicts threshold powers $>2 \mathrm{~mW}$, well beyond the threshold values of the $n=1$ and $n=3$ modes.

We next proceeded to verify the dependence of threshold power on the mechanical quality factor. As both optical and mechanical $Q$ affect threshold, a method was needed which could reduce the mechanical $Q$ factor while leaving the optical $Q$ factor unchanged. To this end, we used a silica microprobe fabricated by heating and stretching a single mode fiber using a $\mathrm{CO}_{2}$ laser. An optical micrograph is shown in the inset of Fig. 14 where the mechanical probe is mounted on a three-axis piezoelectric stack, and positioned above the fiber-taper coupled microtoroid. When the probe was brought into contact with parts of the microstructure exhibiting high-amplitude mechanical oscillations (compare the mode profiles in Fig. 4), a reduction of the mechanical $Q$ factor was observed, while leaving the optical $Q$ factor unaffected. The change in mechanical $Q$-factor was measured by fitting the ESA measured resonances in the subthreshold regime with Lorentzian profiles. Without contact, the mechanical $Q$ was measured to be $\sim 5000$ for the $n=1$ mode, and upon progressive increase in tip pressure (controlled via piezo-electric distance), the mechanical $Q$ could be continuously decreased by nearly two orders of magnitude, down to a value of 50. Upon retrieving the probe, the original $Q$-factor was recovered. The microprobe method thus allowed changing the mechanical $Q$ while leaving the optical modes unperturbed. For each mechanical $Q$, the minimum threshold for the $n=1$ flexural mode (by optimizing both $\Delta \omega$ and K) was measured. Fig. 14 shows the measured minimum threshold for oscillation of $n=1$ mode as a function of mechanical $Q$-factor, clearly revealing the $1 / Q_{m}$ dependence of the oscillation threshold, in agreement with (4) and (7), as well as with the theoretical predictions of Braginsky [26], [27]. This method also provides a technique to prevent parametric oscillation instability for cases where this effect is not desirable.

\section{CONCLUSION}

It should be noted that mechanical oscillations in microstructures can be generated using alternative methods. For example, [28], [29] describe thermally actuated mechanical vibrations of a silicon disk and a silicon cantilever, respectively. In contrast, the long thermal time constant of the toroid structures in the present work ( $\tau_{\text {thermal }}$ is in the order of $5 \mu \mathrm{s}$ [30], [31]) precludes initiation of RF rate oscillations such as those observed here. Also significant is the expected threshold dependence on optical $Q$ for thermally driven instabilities. Because resonator deformation for a thermally driven process (as opposed to radiation pressure) depends on coupled optical power (not circulating power), one expects an inverse quadratic scaling of the threshold power with optical $Q\left(P_{\text {threshold }} \propto Q^{-2}\right)$ for thermally-induced oscillations as opposed to inverse cubic $\left(P_{\text {threshold }} \propto Q^{-3}\right)$ for radiation pressure induced oscillations verified in this study. Excellent agreement of the threshold functional dependence on optical and mechanical $Q$ factors, and precise numerical predictions of the threshold power, provides confirmation that radiation pressure is the excitation mechanism of the observed oscillations.

The work presented here is the first demonstration of the radiation pressure induced parametric instability in optical resonators of any kind. This work demonstrates how vibrational and optical modes of the same cavity structure can act as parametrically coupled oscillators despite of their very different frequencies $\left(\sim 10^{7}\right.$ against $\left.\sim 10^{14} \mathrm{~Hz}\right)$. Realization of this effect in the microscale can be utilized as a platform for studying the parametric instability limitations for the LIGO project.

In small microtoroids (we were also able to observe similar oscillations in silica microspheres), occurrence of these oscillations has been observed with threshold powers well below those of other nonlinear effects such as Raman [32], [33], Kerr parametric oscillation [34] and lasing due to intentional doping of rare earth elements into the microcavities [35]. This clearly suggests that radiation pressure-induced effects can establish a practical limit for miniaturization of optical microcavities. This miniaturization limit can be extended by adding a mechanical damper (or displacement limiter as in Fig. 14) to the cavity structure. Yet, the fundamental nature of circulating light to apply pressure and the general nature of structural stiffness to reduce with miniaturization ${ }^{7}$ suggests that it is likely that all optical cavities are susceptible to these oscillations at various optical powers. The inverse cubic dependence of threshold power suggests that current efforts directed towards realization of higher $Q$ optical microcavities will only tend to accelerate the observation of these oscillations in other microcavity systems, either as a limiting floor in miniaturization, or as a useful new optomechanical nonlinearity.

\footnotetext{
${ }^{7}$ The spring constant of a structure is proportional to its cross-sectional area divided by its length, and hence spring constant decreases with size.
} 

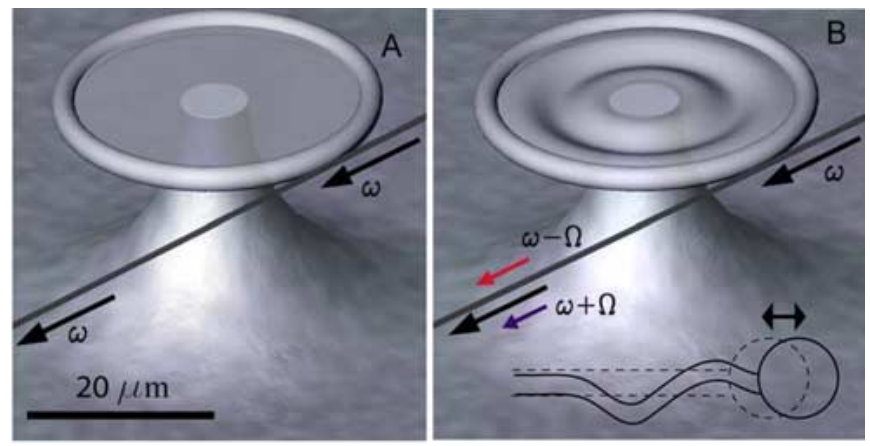

Fig. 15. Panel A illustrates the "below threshold" behavior where the optical pump wave at frequency $\omega$ is not strong enough to induce mechanical oscillations of the microtoroid. Panel B illustrates the "above threshold" case for the $n=3$ vibrational mode. Mechanical oscillation at frequency $\Omega$ creates optical stokes $(\omega-\Omega)$ and anti-Stokes sidebands $(\omega+\Omega)$ in the transmitted pump wave. Inset of panel $\mathrm{B}$ shows the exaggerated cross section of the third order mode and variation of the toroid radius as a result of these oscillations.

Beyond limitations caused by radiation pressure-induced optomechanical coupling, the parametric instability demonstrates a new class of hybrid oscillators where a continuous source of pump laser power (without any type of external feedback system) can generate radio-frequency mechanical vibrations of a micromechanical structure (a rendering of the oscillation process for the $n=3$ mode is shown in Fig. 15). These oscillations imprint onto the optical pump, now an optical carrier for RF frequencies. Realization of this effect undoubtedly benefits applications in RF micromechanical oscillators [36] on a chip, and all-optical frequency reference devices.

Besides the fundamental aspects of this work, the observed coupling of mechanical and optical modes by radiation pressure can find applications in micromechanical and nanomechanical systems [37] for ultrahigh sensitivity measurements of charge [38], displacement, mass, force [39], or biological entities [40]. Equally important, radiation pressure, as observed here, can be used to achieve cooling of mechanical resonator modes [29].

We believe the promising consequences of our recent research results will pave the way for further practical applications of UHQ microcavities and introduce them to an exciting and novel realm of science.

\section{REFERENCES}

[1] V. B. Braginsky, S. E. Strigin, and S. P. Vyatchanin, "Parametric oscillatory instability in Fabry-Perot interferometer," Phys. Lett. A, vol. 287, no. 5-6, pp. 331-333, 2001.

[2] V. B. Braginsky, S. E. Strigin, and S. P. Vyatchanin, "Analysis of parametric oscillatory instability in power recycled LIGO interferometer," Phys. Lett. A., vol. 305, no. 3-4, pp. 111-124, 2002.

[3] A. Abramovici et al., "The laser-interferometer-gravitational-wave observatory," Science, vol. 256, no. 5055, pp. 325-333, 1992.

[4] G. Amelino-Camelia, "Gravity-wave interferometers as quantum-gravity detectors," Nature, vol. 398, no. 6724, pp. 216-218, 1999.

[5] W. Kells and E. D'Ambrosio, "Considerations of parametric instability in Fabry Perot interferometer," Phys. Lett. A, vol. 229, no. 4, pp. 326-330, 2002.

[6] C. Zhao, L. Ju, J. Degallaix, S. Gras, and D. G. Blair, "Parametric instabilities and their control in advanced interferometer gravitational-wave detectors," Phys. Rev. Lett., vol. 94, no. 12, art. no. 121102, 2005.

[7] S. W. Schediwy et al., "An experiment to investigate optical spring parametric instability," Class. Quant. Grav., vol. 21, no. 5, pp. S1253-S12587, 2004.
[8] A. Dorsel, J. D. McCullen, P. Meystre, E. Vignes, and H. Walther, "Optical bistability and mirror confinement induced by radiation pressure," Phys. Rev. Lett., vol. 51, no. 17, pp. 1550-1553, 1983.

[9] V. B. Braginsky, M. L. Gorodetsky, and V. S. Ilchenko, "Quality-factor and nonlinear properties of optical whispering-gallery modes," Phys. Lett. A, vol. 137, no. 7-8, pp. 393-397, 1989.

[10] D. K. Armani, T. J. Kippenberg, S. M. Spillane, and K. J. Vahala, "Ultrahigh- $Q$ toroid microcavity on a chip," Nature, vol. 421, no. 6926, pp. 925$928,2003$.

[11] L. Hilico et al., "Squeezing with chi (3) materials," Appl. Phys. B, Photophys. Laser Chem., vol. 55, pp. 202-209, 1992.

[12] V. Giovannetti, S. Mancini, and P. Tombesi, "Radiation pressure induced Einstein-Podolsky-Rosen paradox," Europhys. Lett., vol. 54, pp. 559-565, 2001.

[13] S. Mancini, V. Giovanetti, D. Vitali, and P. Tombesi, "Entangling macroscopic oscillators exploiting radiation pressure," Phys. Rev. Lett., vol. 88, p. 120401, 2002.

[14] S. Pirandola, S. Mancini, D. Vitali, and P. Tombesi, "Continuous-variable entanglemet and quantum-state teleportation between optical and macroscopic vibrational modes through radiation pressure," Phys. Rev. A, vol. 68, p. $062317,2003$.

[15] W. Marshall, C. Simon, R. Penrose, and D. Bouwmeester, "Towards Quantum Superpositions of a Mirror," Phys. Rev. Lett., vol. 91, p. 130401, 2003.

[16] S. Mancini, D. Vitali, and P. Tombesi, "Scheme for teleportation of quantum states onto a mechanical resonator," Phys. Rev. Lett., vol. 90, p. 137901, 2003.

[17] I. Tittonen et al., "Interferometric measurements of the position of a macroscopic body: Towards observation of quantum limits," Phys. Rev. A, vol. 59, pp. 1038-1044, 1999.

[18] H. Rokhsari, T. J. Kippenberg, T. Carmon, and K. J. Vahala, "Radiationpressure-driven micro-mechanical oscillator," Opt. Exp., vol. 13, no. 14, 2005.

[19] T. J. Kippenberg, H. Rokhsari, T. Carmon, A. Scherer, and K. J. Vahala, "Analysis of radiation-pressure induced mechanical oscillation of an optical microcavity," Phys. Rev. Lett., vol. 95, pp. 033-901, 2005.

[20] T. Carmon, H. Rokhsari, L. Yang, T. J. Kippenberg, and K. J. Vahala, "Temporal behavior of radiation-pressure-induced vibrations of an optical microcavity phonon mode," Phys. Rev. Lett., vol. 94, p. 223902, 2005.

[21] M. Cai, O. Painter, and K. J. Vahala, "Observation of critical coupling in a fiber taper to silica-microsphere whispering gallery mode system," Phys. Rev. Lett., vol. 85, no. 1, pp. 74-77, 2000.

[22] R. M. Shelby, M. D. Levenson, and P. W. Bayer, "Guided acousticwave Brillouin-scattering," Phys. Rev. B., vol. 31, no. 8, pp. 5244$5252,1985$.

[23] M. L. Gorodetsky and V. S. Ilchenko, "Optical microsphere resonators: optimal coupling to high- $Q$ whispering-gallery modes," J. Opt. Soc. Amer. B, Opt. Phys., vol. 16, no. 1, pp. 147-154, 1999.

[24] S. M. Spillane, T. J. Kippenberg, O. J. Painter, and K. J. Vahala, "Ideality in a fiber-taper-coupled microresonator system for application to cavity quantum electrodynamics," Phys. Rev. Lett., vol. 91, no. 4, p. 043902, 2003.

[25] T. J. Kippenberg, S. M. Spillane, and K. J. Vahala, "Modal coupling in traveling-wave resonators," Opt. Lett., vol. 27, no. 19, pp. 1669-1673, 2002.

[26] V. B. Braginsky, M. L. Gorodetsky, and V. S. Ilchenko, "Quality-factor and nonlinear properties of optical whispering-gallery modes," Phys. Lett. $A$, vol. 137, no. 7-8, pp. 393-397, 1989.

[27] V. B. Braginsky, M. L. Gorodetsky, V. S. Ilchenko, and S. P. Vyatchanin, "On the ultimate sensitivity in coordinate measurements," Phys. Lett. A, vol. 179, no. 4-5, pp. 244-248, 1993.

[28] M. Zalalutdinov et al., "Autoparametric optical drive for micromechanical oscillators," Appl. Phys. Lett., vol. 79, pp. 695-697, 2001.

[29] C. H. Metzger and K. Karrai, "Cavity cooling of a microlever," Nature, vol. 432, pp. 1002-1005, 2004.

[30] H. Rokhsari, S. M. Spillane, and K. J. Vahala, "Observation of Kerr nonlinearity in microcavities at room temperature," Opt. Lett., vol. 30, pp. 427-429, 2005

[31] V. S. Ilchenko and M. L. Gorodetsky, "Thermal nonlinear effects in optical whispering gallery microresonators," Laser Phys., vol. 2, pp. 1004-1009, 1992.

[32] S. M. Spillane, T. J. Kippenberg, and K. J. Vahala, "Ultralow-threshold Raman laser using a spherical dielectric microcavity," Nature, vol. 415, pp. 621-623, 2002.

[33] T. J. Kippenberg, S. M. Spillane, B. K. Min, and K. J. Vahala, "Theoretical and experimental study of stimulated and cascaded Raman scattering in 
ultrahigh- $Q$ optical microcavities," IEEE J. Sel. Topics Quantum Electron., vol. 10, no. 5, pp. 1219-1228, Sep./Oct. 2004.

[34] T. J. Kippenberg, S. M. Spillane, and K. J. Vahala, "Kerr-nonlinearity optical parametric oscillation in an ultrahigh- $Q$ toroid microcavity," Phys. Rev. Lett., vol. 93, no. 8, p. 083904, 2004.

[35] L. Yang, D. K. Armani, and K. J. Vahala, "Fiber-coupled Erbium microlasers on a chip," Appl. Phys. Lett., vol. 83, no. 5, pp. 825-826, 2003.

[36] X. M. H. Huang, C. A. Zorman, M. Mehregany, and M. L. Roukes, "Nanodevice motion at microwave frequencies," Nature, vol. 421, no. 6922 , pp. 496-496, 2003.

[37] H. G. Craighead, "Nanoelectromechancial systems," Science, vol. 290, pp. $1532-1535,2000$

[38] A. N. Cleland and M. L. Roukes, "A nanometer-scale mechanical electrometer," Nature, vol. 392, pp. 160-162, 1998.

[39] D. Rugar, R. Budakian, H. J. Mamin, and B. W. Chui, "Single spin detection by magnetic resonance force microscopy," Nature, vol. 430, pp. 329332, 2004.

[40] B. Ilic, Y. Yang, and H. G. Craighead, "Virus detection using anoelectromechanical devices," Appl. Phys. Lett., vol. 85, pp. 2604-2606, 2004.

H. Rokhsari, photograph and biography not available at the time of publication.
T. J. Kippenberg, photograph and biography not available at the time of publication.

T. Carmon, photograph and biography not available at the time of publication.

K. J. Vahala, photograph and biography not available at the time of publication. 